Article

\title{
Improving the Classification Effectiveness of Intrusion Detection by Using Improved Conditional Variational AutoEncoder and Deep Neural Network
}

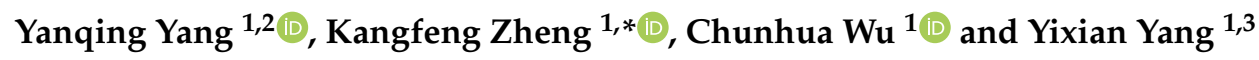 \\ 1 School of Cyberspace Security, Beijing University of Posts and Telecommunications, Beijing 100876, China; \\ qing0991@163.com (Y.Y.); wuchunhua@bupt.edu.cn (C.W.); yxyang@bupt.edu.cn (Y.Y.) \\ 2 College of Information Science and Engineering, Xinjiang University, Urumqi 830046, China \\ 3 Guizhou Provincial Key Laboratory of Public Big Data, Guizhou University, Guiyang 550025, China \\ * Correspondence: zkf_bupt@163.com
}

Received: 18 March 2019; Accepted: 30 May 2019; Published: 2 June 2019

\begin{abstract}
Intrusion detection systems play an important role in preventing security threats and protecting networks from attacks. However, with the emergence of unknown attacks and imbalanced samples, traditional machine learning methods suffer from lower detection rates and higher false positive rates. We propose a novel intrusion detection model that combines an improved conditional variational AutoEncoder (ICVAE) with a deep neural network (DNN), namely ICVAE-DNN. ICVAE is used to learn and explore potential sparse representations between network data features and classes. The trained ICVAE decoder generates new attack samples according to the specified intrusion categories to balance the training data and increase the diversity of training samples, thereby improving the detection rate of the imbalanced attacks. The trained ICVAE encoder is not only used to automatically reduce data dimension, but also to initialize the weight of DNN hidden layers, so that DNN can easily achieve global optimization through back propagation and fine tuning. The NSL-KDD and UNSW-NB15 datasets are used to evaluate the performance of the ICVAE-DNN. The ICVAE-DNN is superior to the three well-known oversampling methods in data augmentation. Moreover, the ICVAE-DNN outperforms six well-known models in detection performance, and is more effective in detecting minority attacks and unknown attacks. In addition, the ICVAE-DNN also shows better overall accuracy, detection rate and false positive rate than the nine state-of-the-art intrusion detection methods.
\end{abstract}

Keywords: intrusion detection; variational inference; improved conditional variational autoencoder; generator network; deep neural network

\section{Introduction}

In recent years, with the rapid development of cloud computing, LoRa, NB-IoT, 5G communication and artificial intelligence technologies, the internet of things (IoT) technology has also ushered in a boom-like development, and hundreds of millions of devices are connected to the Internet of Things. However, because many IoT nodes collect and store large amounts of user privacy data, IoT systems have become an ideal target for cyber attackers, and attacks on the Internet of Things are increasing [1,2]. Gemalto's IoT security report shows that more than half of companies still can't find out whether they have suffered IoT vulnerability attacks. In addition, the report surveyed 950 IT and business decision makers and found that only $59 \%$ of companies encrypted all IoT-related data [3]. The popularity of IoT technology and the intelligence of devices have brought great convenience to people, but the use of new technologies and intelligent devices has also brought new security and privacy risks. For example, 
on 29 January 2018, the top three banks (ABN AMRO, ING Bank, Rabobank) in the Netherlands were attacked by distributed denial of service (DDoS), blocking access to websites and internet banking services [4]. In February 2018, the Pyeongchang Winter Olympics in South Korea suffered a cyber attack, which caused the live broadcast to be interrupted [5]. Therefore, maintaining the security of the IoT system is becoming the focus of successful deployment of the IoT network, and detecting intruders is an important step to ensure the security of the IoT network. Intrusion detection is one of several security mechanisms to manage security intrusions [6]. It monitors network traffic for abnormal or suspicious activity and issues alerts when such activity is discovered. Intrusion detection system (IDS) can be classified into host-based intrusion detection systems (HIDS) and network-based intrusion detection system (NIDS). In this paper, we study a network-based intrusion detection system. We studied the use of generation models and deep learning techniques to build intrusion detection classifiers to detect a great variety of attacks, such as DoS (denial of service), probe, U2R (user to root), R2L (remote to local), worms, shellcode, backdoor, reconnaissance, generic attacks, etc.

Many researchers have introduced more and more innovative approaches to detect intrusions in recent years, including anomaly detection methods, shallow learning methods, deep learning methods, and ensemble methods. The anomaly detection methods calculate the distribution of normal network data and define any data that deviates from the normal distribution as an anomaly, such as Bayesian models [7,8], the Cluster algorithms (K-Means, spectral clustering, DBSCAN, etc.) [7], self-organizing map (SOM) [9], Gaussian mixture model (GMM) [10], and one-class SVM [11]. Shallow learning methods use the selected features to build a classifier to detect intrusions, such as support vector machines (SVM) [12], decision tree (DT) [13], and k-nearest neighbor (KNN) [14]. Deep learning methods can automatically extract features and perform classification, such as AutoEncoder $[15,16]$, deep neural network (DNN) [17], deep belief network (DBN) [18-21], and recurrent neural network (RNN) [22]. The last category uses various ensemble and hybrid techniques to improve detection performance, including bagging [23], boosting [24], stacking [25], and combined classifier methods [26].

Deep learning is a data representation and learning method based on machine learning, which has become a hot research topic. It can automatically extract high-level latent features without manual intervention [27]. Deep learning is widely applied in many fields of artificial intelligence, including speech processing, computer vision, natural language processing and so on. Moreover, deep learning has been applied to network security detection $[28,29]$. However, there are still many problems with intrusion detection systems. First, different types of network traffic in a real network environment are imbalanced, and network intrusion records are less than normal records. The classifier is biased towards the more frequently occurring records, which reduces the detection rate of minority attacks such as R2L and worms attacks. Second, because of the high dimension of network traffic, the feature selection method in many intrusion models is first considered as one of the pre-processing steps [30], such as principal component analysis (PCA) and chi-square feature selection. However, these feature selection methods rely heavily on manual feature extraction, mainly through experience and luck, and these algorithms are not effective enough. Third, due to the large network traffic and complex structure, the traditional classifier algorithm is difficult to achieve high detection rate. Fourth, the network operating environment and structure in the real world are changing, for example, the Internet of Things and cloud services are widely used and various new attacks are emerging. Since many unknown attacks do not appear in the training dataset, traditional intrusion detection methods usually perform poorly in detecting unknown attacks.

Taking into account the above factors, we propose a novel intrusion detection method called ICVAE-DNN, which combines improved conditional variational AutoEncoder (ICVAE) with DNN. The variational AutoEncoder (VAE) is an important generation model consisting of an encoder (a recognition network) and a decoder (a generator network) that use deep neural networks to characterize the distribution of data and latent variables, which was proposed by Kingma et al. [31] in 2013. VAE can generate samples, but it is not possible to generate some specific samples based on the labels. Therefore, CVAE was developed by Kingma et al. [32] in 2014. The CVAE is an extension 
of VAE [33]. It embeds a one-hot encoded label vector in the encoder and decoder, and converts unsupervised training mode into supervised training mode. CVAE not only automatically extracts high-level features and reduces the dimensions of network features, but also generate new attack samples of the specified categories. In order to initialize the weight of the DNN hidden layers using the CVAE encoder, we have improved CVAE by embedding intrusion tags only in the decoder, but not in the encoder, named ICVAE.

This paper has the following main contributions. First, we use ICVAE to learn the distribution of complex traffic and classes through supervised learning. The network parameters of ICVAE encoder are used to initialize the weight of DNN hidden layers. Second, latent variables with Gaussian noise and specified labels are fed into the trained ICVAE decoder (generating network) to generate specific new attack records, so as to balance the training data and increase the diversity of training samples, thus improving the detection rate of minority attacks and unknown attacks. Third, DNN is used to automatically extract high-level features, and adjust network weights by back propagation and fine-tuning to better address the classification problem of complex, large-scale and non-linear network traffic. Finally, the proposed model is evaluated on the NSL-KDD [34,35] and UNSW-NB15 [36-38] datasets. Compared with the well-known classification methods, the proposed model not only reaches better overall accuracy, recall, and false positive rate, but also achieves higher detection rate in minority attacks and unknown attacks.

The remainder of this paper is organized as follows. The related works are introduced in Section 2. Section 3 describes the ICVAE and DNN algorithms. Section 4 proposes a novel intrusion detection model and shows in detail how the model works. Section 5 demonstrates the experimental details and results. Finally, Section 6 provides some conclusions and further work.

\section{Related Works}

Although there are CVAE-related work in other fields, there is no report on the combination of ICVAE and DNN for intrusion detection. Kawachi et al. [39] employed a VAE for supervised anomaly detection. Sun et al. [40] used a VAE to learn sparse representations for anomaly detection. Chandy et al. [41] used VAE as a deep generation model to simulate network attack detection problems. Osada et al. [42] employed VAE as a semi-supervised learning for intrusion detection. They use VAE to detect intrusions, not CVAE. Lopez-Martin et al. [16] used conditional VAE (CVAE) to build an ID-CVAE classifier to perform classification and feature recovery. The ID-CVAE uses the reconstructed test data and the nearest neighbor method based on the Euclidean distance to classify the test samples. However, our proposed model not only generates data according to categories, but also uses DNN classifier to perform classification.

The deep learning method integrates high-level feature extraction and classification tasks, overcomes some limitations of shallow learning, and further promotes the progress of intrusion detection systems. Recently, deep learning models have been widely used in the field of intrusion detection. Stacked AutoEncoders are used to detect attacks in IEEE 802.11 networks with an overall accuracy of $98.60 \%$ [43]. Ma et al. [44] presented a hybrid method combining spectral clustering and deep neural networks to detect attacks with an overall accuracy of $72.64 \%$ on the NSL-KDD dataset. The gated recurrent unit recurrent neural network (GRU-RNN) was used to build an intrusion detection system in an software defined network (SDN) with an accuracy of 89\% [45]. Shone et al. [15] employed a stacked non-symmetric AutoEncoder and random forest (RF) to detect attacks. Muna et al. [46] proposed an anomaly detection technique for internet industrial control systems (IICSs) based on the deep learning model, which used deep auto-encoder for feature extraction and deep feedforward neural network for classification. Tamer et al. [20] employed the restricted Boltzmann machine (RBM) to classify normal and abnormal network traffic. Imamverdiyev [18] used the multilayer deep Gaussian-Bernoulli RBM method to detect DoS attacks with an accuracy of $73.23 \%$ on the NSL-KDD dataset. 
The above intrusion detection evaluation results are very encouraging, but these classification techniques still have detection defects, low detection rate for unknown attacks and high false positive rate for minority attacks. In order to overcome these classification problems, this paper uses ICVAE decoder to generate new attack samples according to the specified intrusion categories, thereby improving the detection rate of unknown attacks and minority attacks. ICVAE encoder automatically learns the potential representation of input data and reduces the dimensions of features. Furthermore, the ICVAE encoder is used to initialize the weight of DNN hidden layers. Finally, it is easier for DNN to achieve global optimization by back propagation and fine tuning network parameters.

\section{Background}

\subsection{Variational AutoEncoder (VAE)}

Variational AutoEncoder (VAE) is an important generation model consisting of an encoder network $Q_{\phi}(Z \mid X)$ and a decoder network $P_{\theta}(X \mid Z)$, as shown in Figure 1. VAE can learn approximate inference and can be trained using gradient descent method. The encoder network with parameters $\phi$ learns an efficient compression of the data into this lower-dimensional space, which maps data $X$ into a continuous latent variable $Z$. The decoder network with parameters $\theta$ uses the latent variable to generate data, which maps $Z$ to a reconstructed data $\hat{X}$. Here we use deep neural networks to construct the encoder and decoder with parameters $\theta$ and $\phi$, respectively.

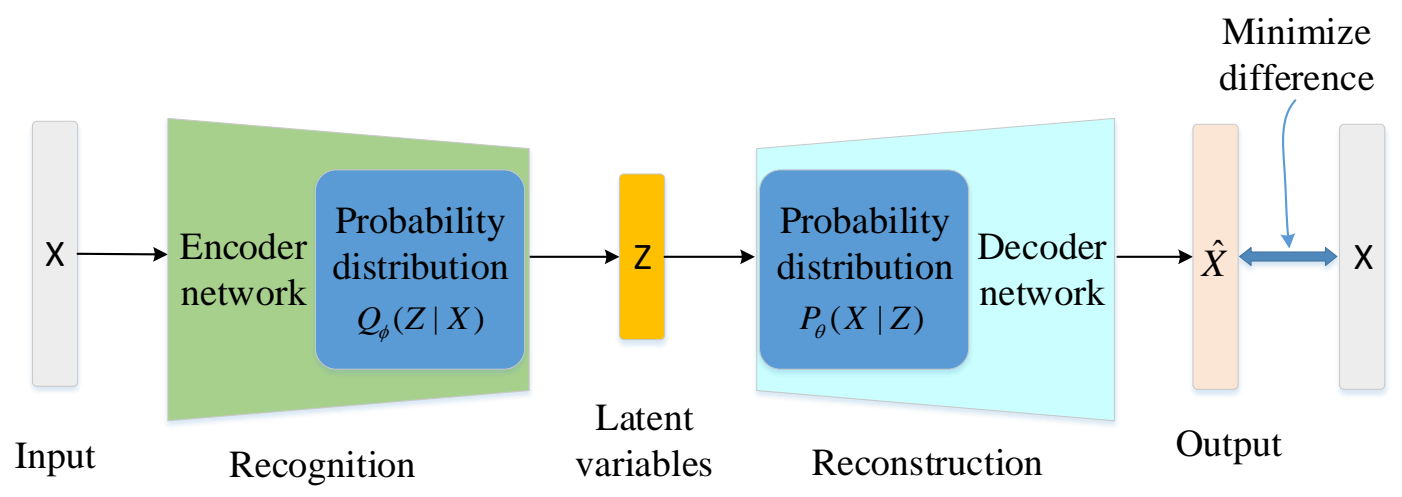

Figure 1. Variational AutoEncoder (VAE) architecture.

The core idea of VAE is to use the probability distribution $P(X)$ to sample data points that match this distribution, where $X$ represents a random variable of the data. The goal of VAE is to reconstruct the input data as much as possible, that is, to maximize the log likelihood probability of $P(X)[31,47]$, as follows:

$$
\begin{aligned}
\log P(X) & =\mathbb{E}[\log P(X \mid Z)]-D_{K L}[Q(Z \mid X) \mid P(Z)]+D_{K L}[Q(Z \mid X)|| P(Z \mid X)] \\
& \geq \mathbb{E}[\log P(X \mid Z)]-D_{K L}[Q(Z \mid X)|| P(Z)]
\end{aligned}
$$

Here the variational lower bound objective $[31,47]$ is defined as follows:

$$
\mathcal{L}(\theta, \phi ; X)=\mathbb{E}[\log P(X \mid Z)]-D_{K L}[Q(Z \mid X) \| P(Z)] .
$$

$\mathcal{L}$ is defined as the variation lower bound, which is called the VAE objective function. The first term in Equation (2) represents the reconstruction loss. It encourages the decoder to learn to reconstruct the input data. The second item in Equation (2) uses KL (Kullback-Leibler) divergence to minimize the difference between the encoder's distribution $Q(Z \mid X)$ and the prior distribution $P(Z)$, that is to say, the learned distribution $Q(Z \mid X)$ is similar to the prior distribution $P(Z)$. Therefore, the goal of training VAE is to maximize the data generation probability $\log P(X \mid Z)$ and minimize the difference between 
the learned distribution $Q(Z \mid X)$ and the true prior distribution $P(Z)$. In other words, the goal of training VAE is to maximize the variational lower bound $\mathcal{L}$.

\subsection{Improved Conditional Variational AutoEncoder (ICAVE)}

Conditional Variational AutoEncoder (CVAE) is an extension of VAE [33], modeled by conditioning the encoder and decoder to class $Y$, as shown in Figure 2. The encoder $Q(Z \mid X, Y)$ is now conditional on two variables $X$ and $Y$, and the decoder $P(X \mid Z, Y)$ is now conditioned on two variables $Z$ and $Y$.

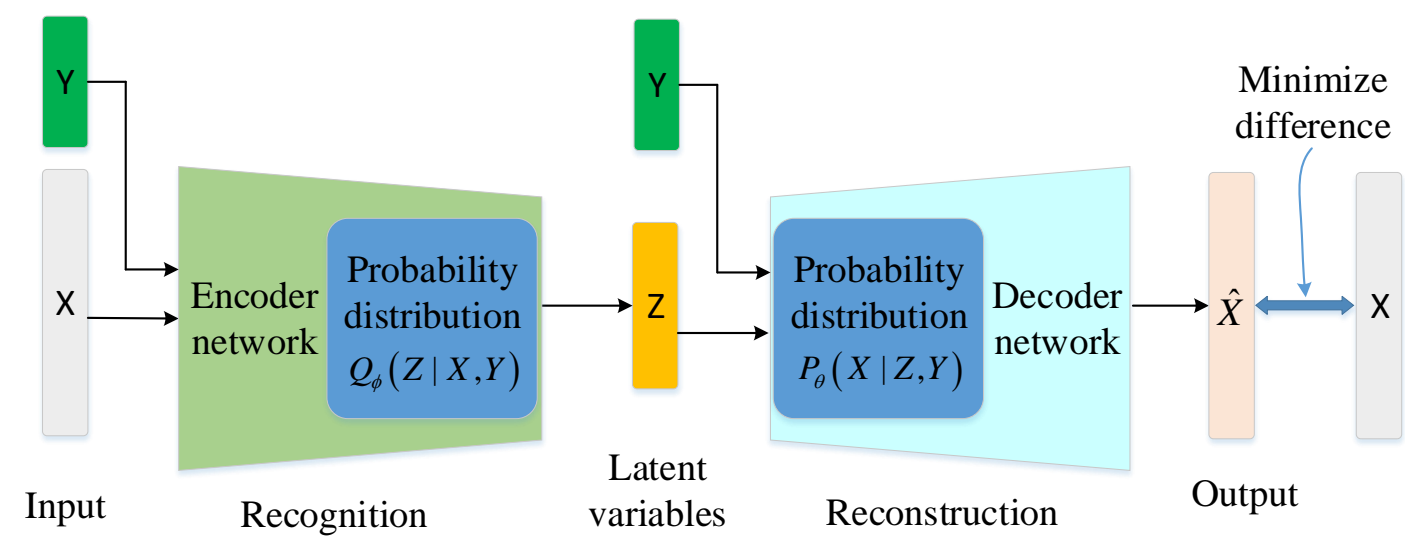

Figure 2. Conditional variational AutoEncoder (CVAE) architecture.

Hence, the variational lower bound objective of CVAE [32,33] is defined as follows:

$$
\log P(X \mid Y)-D_{K L}[Q(Z \mid X, Y) \| P(Z \mid X, Y)]=\mathbb{E}[\log P(X \mid Z, Y)]-D_{K L}[Q(Z \mid X, Y) \| P(Z \mid Y)]
$$

The conditional probability distributions of CVAE encoder and decoder are related to class label $Y$. In order to use the encoder network of CVAE to initialize the network parameters of DNN, we improve the CVAE structure to embed class label $Y$ only in the decoder network. The architecture of ICVAE is shown in Figure 3. The decoder is now conditioned to two variables $Z$ and $Y$ whereas the encoder is now conditioned to one variable $X$.

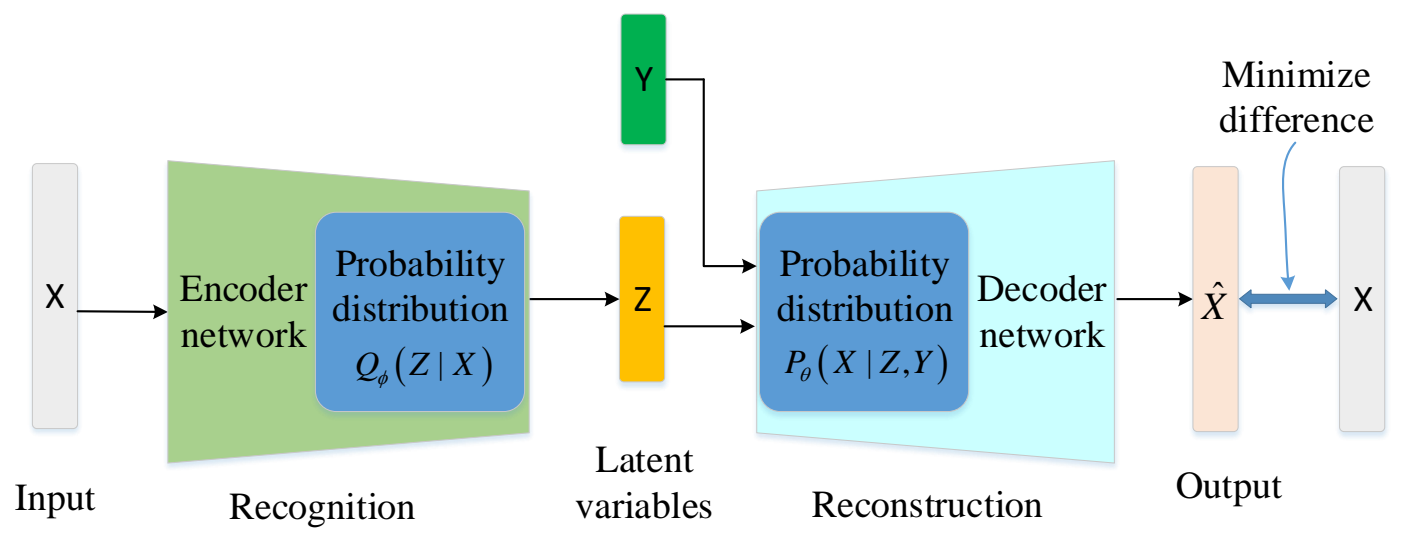

Figure 3. Improved conditional variational AutoEncoder (ICVAE) architecture.

ICVAE is composed of encoder network $Q_{\phi}(Z \mid X)$ and decoder network $P_{\theta}(X \mid Z, Y)$. In the decoder, class labels are used as an extra input, so that the decoder probability distribution is conditional on the latent variable $Z$ and class label $Y$, while the encoder does not contain the class label $Y$. When decoding, the latent variable $Z$ and the label $Y$ are connected and fed to the decoder, 
thus new attack samples of the specified class are generated. Hence, the variational lower bound of ICVAE is defined as follows:

$$
\log P(X \mid Y)-D_{K L}[Q(Z \mid X) \| P(Z \mid X, Y)]=\mathbb{E}[\log P(X \mid Z, Y)]-D_{K L}[Q(Z \mid X) \| P(Z \mid Y)] .
$$

Here the variational lower bound objective of ICVAE is rewritten as:

$$
\mathcal{L}(\theta, \phi ; X, Y)=\mathbb{E}[\log P(X \mid Z, Y)]-D_{K L}[Q(Z \mid X) \| P(Z \mid Y)] .
$$

$\mathcal{L}(\theta, \phi ; X, Y)$ in Equation (5) consists of two parts: a log reconstruction likelihood $\mathbb{E}[\log P(X \mid Z, Y)]$ and a KL divergence $D_{K L}[Q(Z \mid X) \| P(Z \mid Y)]$. The first term is to reconstruct $X$ by using the conditional probability distribution $P(X \mid Z, Y)$ and the second term uses the KL divergence metric to characterize the encoder distribution $Q(Z \mid X)$ approximating the prior distribution $P(Z \mid Y)$. In ICVAE, we try to maximize the the variational lower bound objective $\mathcal{L}(\theta, \phi ; X, Y)$. In this model, We use the class label as our conditional variable $Y$. Obviously we could sample $Z$ from a multivariate standard normal distribution $N(0, I)$. By changing the value of $Y$, such as the attack class in the NSL-KDD dataset, ICVAE's decoder $P(X \mid Z, Y)$ can generate new attack samples of the specified category.

\section{The Proposed Intrusion Detection Framework}

The framework of the proposed ICVAE-DNN is shown in Figure 4. ICVAE-DNN consists of three main phases: (1) training ICVAE, where the training samples are used to train the ICVAE, and the reconstruction loss for each training data sample is stored according to the attack class; (2) generating new attacks, where the ICVAE decoder generates new attack samples based on specified classes, and each newly generated attack sample is merged into the original training data set under the condition that the class reconstruction loss is satisfied; (3) detecting attacks, where the ICVAE decoder is used to initialize the weight of the DNN hidden layers, the merged training data set is used to train the DNN classifier, and the trained DNN classifier is used to detect attacks on the testing data set.

\subsection{Training ICVAE}

The input value of ICVAE must be a real vector, so each symbol feature in the intrusion detection dataset is first converted to a numerical feature. For example, the NSL-KDD [34,35] dataset contains 3 symbol features and 38 numerical features, and the UNSW-NB15 [36-38] dataset contains 3 symbol features and 39 numerical features. All symbol features are transformed to a binary one-hot encoding. The NSL-KDD and UNSW-NB15 datasets are converted into 122-dimensional and 196-dimensional features, respectively. The structure of ICVAE is composed of an encoder and a decoder, as shown in Figure 4. For the encoder $Q(Z \mid X)$, we use a multivariate Gaussian distribution as the $Q(Z \mid X)$ distribution. For the decoder $P(X \mid Z, Y)$, we use a multivariate Bernoulli distribution to fit $P(X \mid Z, Y)$. The output of the decoder network is reconstructed data, which is the predicted probability.

We use the min-max normalization method to scale all data $X$ to $[0,1]$. After preprocessing all the data in the intrusion detection dataset, we train the ICVAE to optimize the loss of the encoder $\theta$ and the decoder $\phi$ by using the balanced sampling via label shuffling [48] and Adam [49] optimization algorithm. The ICVAE loss is composed of a reconstruction loss and a KL loss. The KL loss uses the variational inference method to approximate the distribution $P(Z \mid Y)$ with the deep neural network $Q(Z \mid X)$, so the ICVAE may have a KL-vanishing problem. ICVAE directly compares the difference between the reconstructed attack and the original attack through the encoding and decoding steps. However, the new attack samples generated by ICVAE decoder may differ greatly, and the newly generated samples may deviate from the original attack space distribution. In order to better select the newly generated attack samples, we calculate the reconstruction loss of each training sample based on the class and then use the maximum reconstruction loss for each class as the screening criteria. 


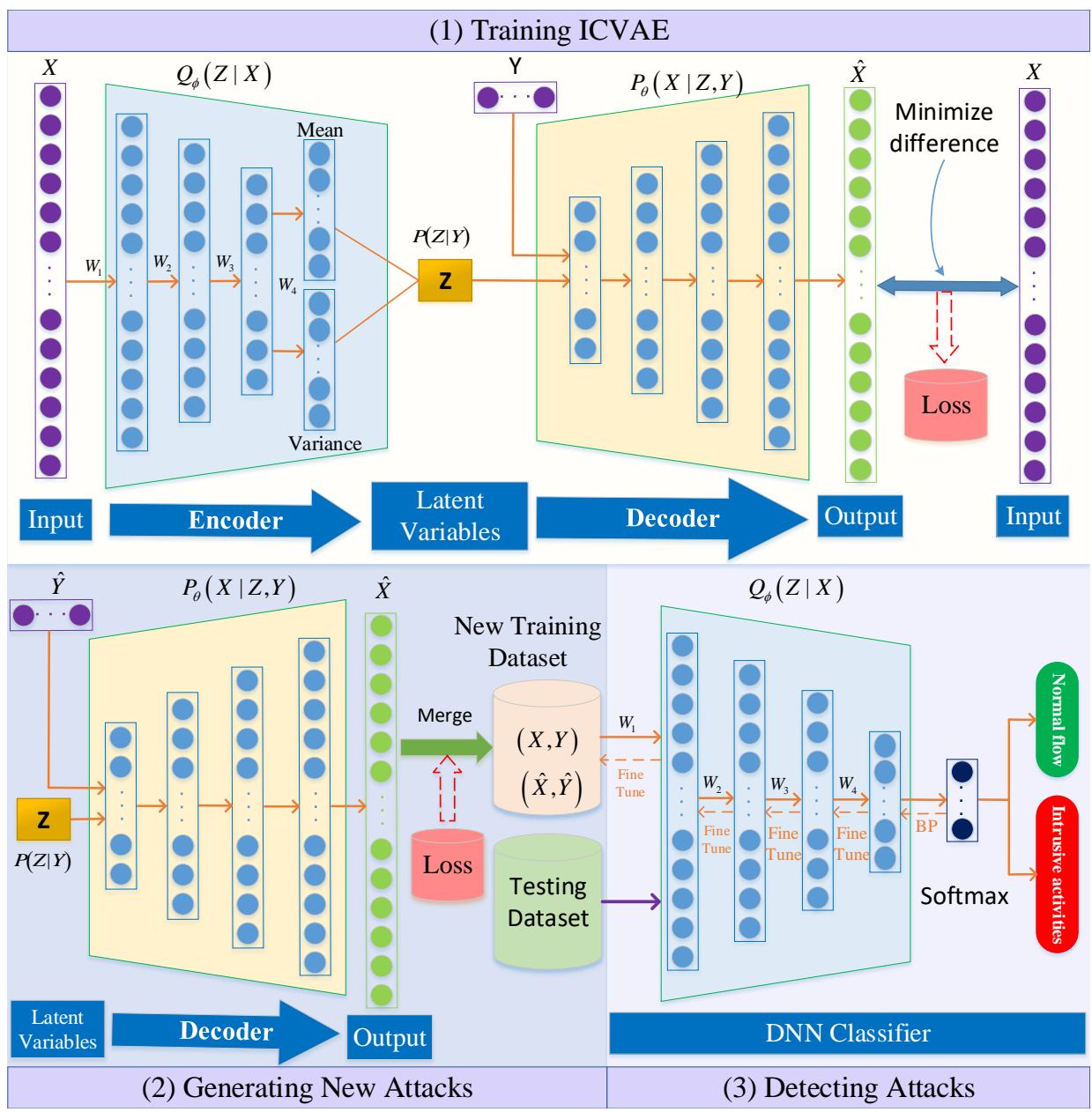

Figure 4. The proposed intrusion detection framework.

We assume that the decoder $P\left(x_{i} \mid z, y\right)$, (where $\left.i=1, \cdots, n\right)$ obeys the Bernoulli distribution, i.e.,

$$
\begin{aligned}
& P(x=1 \mid z, y)=\alpha_{z, y}, \\
& P(x=0 \mid z, y)=1-\alpha_{z, y} .
\end{aligned}
$$

For an observation, the likelihood is:

$$
L=\alpha_{z, y}^{x} \cdot\left(1-\alpha_{z, y}\right)^{1-x}
$$

The decoder output is a parameter of Bernoulli distribution, that is, $\alpha_{z, y}=\operatorname{Decoder}(z, y)=\hat{x}$. Then the negative log likelihood is:

$$
-\log L=-[x \cdot \log (\hat{x})+(1-x) \cdot \log (1-\hat{x})]
$$

It is obvious that the negative log likelihood in Equation (9) is the cross entropy. We use this cross entropy as the reconstruction loss of the decoder. After each training sample $\left(x_{i}, y_{i}\right)$ is fed into the trained ICVAE, the reconstruction loss $l_{i}\left(x_{i}, y_{i}\right)$ can be calculated as follows:

$$
l_{i}\left(x_{i}, y_{i}\right)=-\left[x_{i} \cdot \log \left(\hat{x}_{i}\right)+\left(1-x_{i}\right) \log \left(1-\hat{x}_{i}\right)\right]
$$

The maximum reconstruction loss $\max L_{j}$ of the $j$-th class is written as follows:

$$
\max L_{j}=k * \max \left\{l_{i}\left(x_{i}, y_{i}\right)\right\}, \text { for each } y_{i} \in \text { class } \mathrm{j} \text {, }
$$


where $k$ represents the maximum reconstruction loss scaling factor, typically $k$ is 1.0 .

\subsection{Generating New Attacks}

For the encoder $Q(Z \mid X)$, we use a multivariate Gaussian distribution as the $Q(Z \mid X)$ distribution. For the decoder $P(X \mid Z, Y)$, we define the multivariate standard normal distribution $N(0, I)$ as the prior distribution $P(Z \mid Y)$, that is, $Z \sim N(0, I)$. We can sample a latent variable $z$ from $N(0, I)$ under a specified label $\hat{y}$ and feed it into the trained decoder to generate a new attack sample $(\hat{x}, \hat{y})$. Assuming that the new attack sample belongs to class $j$, i.e., $\hat{y} \in$ class $j$, the generated sample $(\hat{x}, \hat{y})$ is fed into the trained ICVAE and the reconstruction loss $l(\hat{x}, \hat{y})$ is calculated according to Equation (10). Then, we compare the reconstruction loss $l(\hat{x}, \hat{y})$ with the maximum loss $\max L_{j}$ of the corresponding class $j$. If $l(\hat{x}, \hat{y})<\max L_{j}$, the newly generated sample is merged into the original training set $S$, otherwise the sample is discarded. The newly generated attack samples are merged into the original training set according to the following criteria:

$$
\text { if } \hat{y} \in \text { class } \mathrm{j} \text { and } l(\hat{x}, \hat{y}) \leq \max L_{j} \text {, then } S=S \cup\{\hat{x}, \hat{y}\} \text {, else } S=S \text {. }
$$

\subsection{Detecting Attacks}

We employ DNN to detect attacks. DNN is a six-layer feedforward deep neural network. The activation function of all hidden layers in DNN is ReLU6 [50], and the activation function of the output layer in DNN is softmax. The network structure of DNN hidden layers is exactly the same as that of ICVAE encoder. ICVAE encoder can automatically extract high-level features, so the weight of the trained ICVAE encoder is used to initialize the weight of DNN hidden layers, then the merged training data set is used to fine tune DNN classifier, and the DNN classifier is optimized by Adam [49] algorithm. Finally, test samples are fed into the trained DNN classifier to detect attacks.

The proposed intrusion detection model is detailed in Algorithm 1.

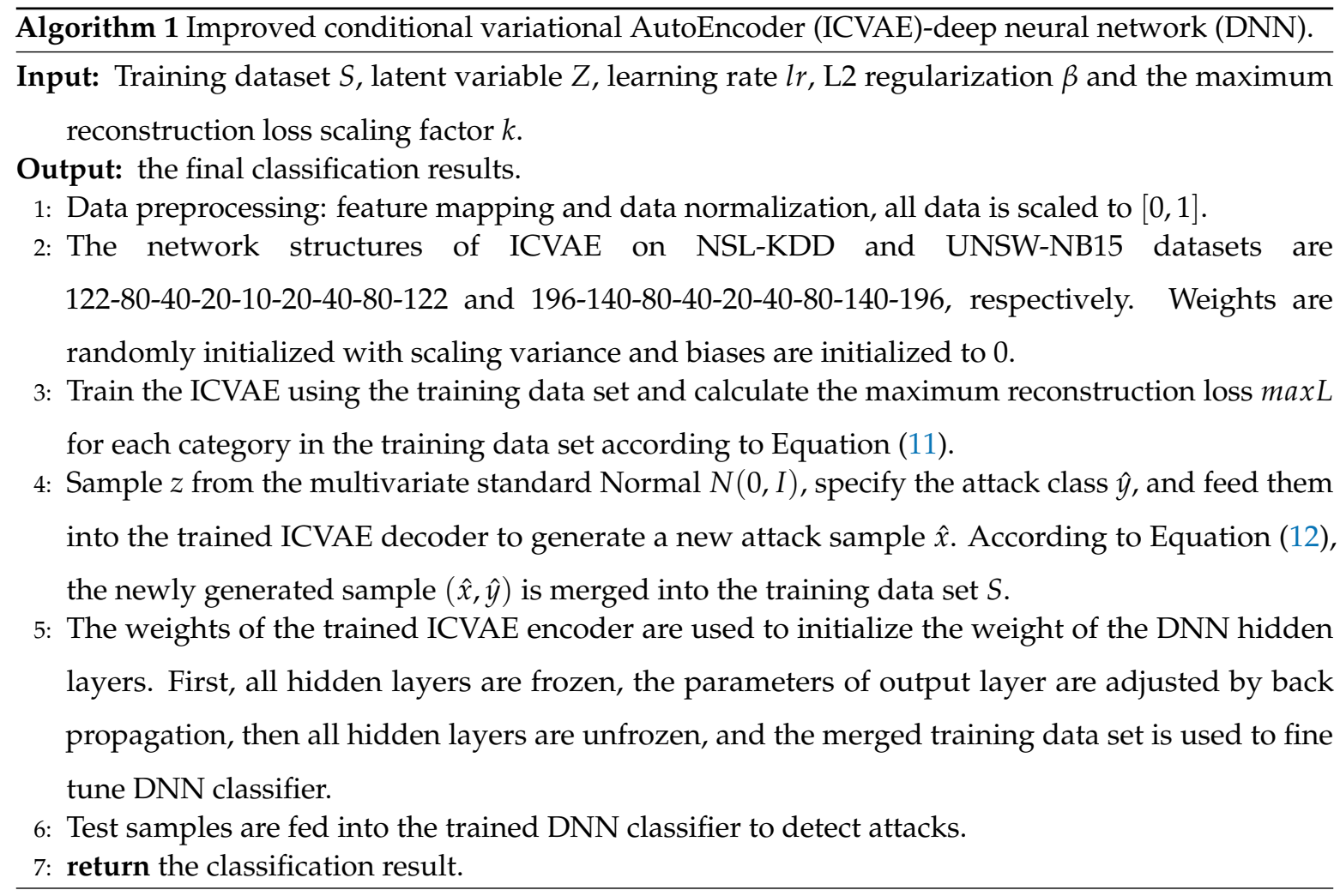




\section{Experimental Results and Analysis}

\subsection{Performance Evaluation}

We use six commonly metrics to evaluate intrusion detection performance, including accuracy, detection rate (DR), precision, recall, false positive rate (FPR), and F1-score. Table 1 shows the confusion matrix consisting of true positive (TP), true negative (TN), false positive (FP), and false negative (FN). TP and TN indicate that the attack and normal records are correctly classified, respectively; FP represents a normal record that is incorrectly predicted as an attack; FN represents an attack record that is incorrectly classified as a normal record.

Table 1. The confusion matrix.

\begin{tabular}{ccc}
\hline & Predicted Attack & Predicted Normal \\
\hline Actual attack & TP & FN \\
Actual normal & FP & TN \\
\hline
\end{tabular}

The accuracy, DR, precision, recall and FPR are defined as follows:

$$
\begin{gathered}
\text { Accuracy }=\frac{\mathrm{TP}+\mathrm{TN}}{\mathrm{TP}+\mathrm{TN}+\mathrm{FP}+\mathrm{FN}^{\prime}}, \\
\mathrm{DR}=\frac{\mathrm{TP}}{\mathrm{TP}+\mathrm{FN}^{\prime}} \\
\text { Precision }=\frac{\mathrm{TP}}{\mathrm{TP}+\mathrm{FP}^{\prime}} \\
\text { Recall }=\frac{\mathrm{TP}}{\mathrm{TP}+\mathrm{FN}^{\prime}} \\
\mathrm{FPR}=\frac{\mathrm{FP}}{\mathrm{FP}+\mathrm{TN}} .
\end{gathered}
$$

The F1-score is a measure of recall and precision using harmonic mean. Compared with the accuracy, F1-score is more suitable for evaluating the detection performance of imbalanced samples. It can be defined:

$$
\text { F1-score }=\frac{2(\text { Precision } * \text { Recall })}{\text { Precision }+ \text { Recall }}=\frac{2 \mathrm{TP}}{2 \mathrm{TP}+\mathrm{FP}+\mathrm{FN}} .
$$

\subsection{Datasets}

Currently, the most common data sets used to evaluate the performance of network intrusion detection systems in the literature are the NSL-KDD [34,35] and UNSW-NB15 [36-38] data sets. Therefore, we selected the NSL-KDD and UNSW-NB15 data sets to validate the proposed model.

\subsubsection{NSL-KDD Dataset}

The NSL-KDD is derived from the raw KDD Cup 99 [51,52] dataset presented by Tavallaee et al. [52]. The NSD-KDD dataset removes duplicate and redundant records in the KDD Cup 99 dataset and is more suitable for evaluating the performance of intrusion detection systems. There are five classes in the NSL-KDD data set, one normal and four attacks, namely, Probe, denial of service (DoS), user to root (U2R), and remote to local (R2L).

The NSL-KDD dataset is imbalanced, with fewer U2R and R2L records. We used two data sets in the NSL-KDD dataset to evaluate intrusion detection performance: KDDTrain+_20Percent.txt (A 20\% subset of the full training set), KDDTest+.txt, and KDDTest-21.txt (A subset of the full test set, excluding records of difficulty level 21). In our experiments, KDDTrain+_20Percent is used as a training set, and KDDTest+ and KDDTest-21 are used as test sets. Table 2 shows the number of records 
for each category on the NSL-KDD dataset. As can be seen from Table 2, approximately 50\% of the unknown attacks in the testing dataset did not appear in the training dataset.

Table 2. The class distribution of the NSL-KDD dataset.

\begin{tabular}{|c|c|c|c|c|c|c|}
\hline \multirow{3}{*}{ Category } & \multirow{2}{*}{\multicolumn{2}{|c|}{$\begin{array}{c}\text { Training Dataset } \\
\text { KDDTrain+_20Percent }\end{array}$}} & \multicolumn{4}{|c|}{ Testing Dataset } \\
\hline & & & \multicolumn{2}{|c|}{ KDDTest+ } & \multicolumn{2}{|c|}{ KDDTest-21 } \\
\hline & Attack & Count & Attack & Count & Attack & Count \\
\hline Normal & normal & 13,449 & normal & 9711 & normal & 2152 \\
\hline Subtotal & & 13,449 & & 9711 & & 2152 \\
\hline Probe & $\begin{array}{l}\text { ipsweep } \\
\text { satan } \\
\text { portsweep } \\
\text { nmap }\end{array}$ & $\begin{array}{l}710 \\
691 \\
587 \\
301\end{array}$ & $\begin{array}{l}\text { ipsweep } \\
\text { satan } \\
\text { portsweep } \\
\text { nmap } \\
\text { saint } \\
\text { mscan }\end{array}$ & $\begin{array}{c}141 \\
735 \\
157 \\
73 \\
319 \\
996 \\
\end{array}$ & $\begin{array}{l}\text { ipsweep } \\
\text { satan } \\
\text { portsweep } \\
\text { nmap } \\
\text { saint } \\
\text { mscan } \\
\end{array}$ & $\begin{array}{c}141 \\
727 \\
156 \\
73 \\
309 \\
996 \\
\end{array}$ \\
\hline Subtotal & & 2289 & & 2421 & & 2402 \\
\hline DoS & $\begin{array}{l}\text { neptune } \\
\text { smurf } \\
\text { back } \\
\text { teardrop } \\
\text { pod } \\
\text { land }\end{array}$ & $\begin{array}{c}8282 \\
529 \\
196 \\
188 \\
38 \\
1\end{array}$ & $\begin{array}{l}\text { neptune } \\
\text { smurf } \\
\text { back } \\
\text { teardrop } \\
\text { pod } \\
\text { land } \\
\text { apache2 } \\
\text { mailbomb } \\
\text { processtable } \\
\text { udpstorm }\end{array}$ & $\begin{array}{c}4657 \\
665 \\
359 \\
12 \\
41 \\
7 \\
737 \\
293 \\
685 \\
2\end{array}$ & $\begin{array}{l}\text { neptune } \\
\text { smurf } \\
\text { back } \\
\text { teardrop } \\
\text { pod } \\
\text { land } \\
\text { apache2 } \\
\text { mailbomb } \\
\text { processtable } \\
\text { udpstorm }\end{array}$ & $\begin{array}{c}1579 \\
627 \\
359 \\
12 \\
41 \\
7 \\
737 \\
293 \\
685 \\
2\end{array}$ \\
\hline Subtotal & & 9234 & & 7458 & & 4342 \\
\hline U2R & $\begin{array}{l}\text { buffer_overflow } \\
\text { rootkit } \\
\text { loadmodule }\end{array}$ & $\begin{array}{l}6 \\
4 \\
1\end{array}$ & $\begin{array}{l}\text { buffer_overflow } \\
\text { rootkit } \\
\text { loadmodule } \\
\text { perl } \\
\text { httptunnel } \\
\text { ps } \\
\text { sqlattack } \\
\text { xterm }\end{array}$ & $\begin{array}{c}20 \\
13 \\
2 \\
2 \\
133 \\
15 \\
2 \\
13\end{array}$ & $\begin{array}{l}\text { buffer_overflow } \\
\text { rootkit } \\
\text { loadmodule } \\
\text { perl } \\
\text { httptunnel } \\
\text { ps } \\
\text { sqlattack } \\
\text { xterm }\end{array}$ & $\begin{array}{c}20 \\
13 \\
2 \\
2 \\
133 \\
15 \\
2 \\
13\end{array}$ \\
\hline Subtotal & & 11 & & 200 & & 200 \\
\hline R2L & $\begin{array}{l}\text { guess_passwd } \\
\text { warezmaster } \\
\text { imap } \\
\text { multihop } \\
\text { phf } \\
\text { ftp_write } \\
\text { spy } \\
\text { warezclient }\end{array}$ & $\begin{array}{c}10 \\
7 \\
5 \\
2 \\
2 \\
1 \\
1 \\
181\end{array}$ & $\begin{array}{l}\text { guess_passwd } \\
\text { warezmaster } \\
\text { imap } \\
\text { multihop } \\
\text { phf } \\
\text { ftp_write } \\
\text { named } \\
\text { sendmail } \\
\text { xlock } \\
\text { xsnoop } \\
\text { worm } \\
\text { snmpgetattack } \\
\text { snmpguess }\end{array}$ & $\begin{array}{c}1231 \\
944 \\
1 \\
18 \\
2 \\
3 \\
17 \\
14 \\
9 \\
4 \\
2 \\
178 \\
331\end{array}$ & $\begin{array}{l}\text { guess_passwd } \\
\text { warezmaster } \\
\text { imap } \\
\text { multihop } \\
\text { phf } \\
\text { ftp_write } \\
\text { named } \\
\text { sendmail } \\
\text { xlock } \\
\text { xsnoop } \\
\text { worm } \\
\text { snmpgetattack } \\
\text { snmpguess }\end{array}$ & $\begin{array}{c}1231 \\
944 \\
1 \\
18 \\
2 \\
3 \\
17 \\
14 \\
9 \\
4 \\
2 \\
178 \\
331\end{array}$ \\
\hline Subtotal & & 209 & & 2754 & & 2754 \\
\hline Total & & 25,192 & & 22,544 & & 11,850 \\
\hline
\end{tabular}




\subsubsection{UNSW-NB15 Dataset}

The UNSW-NB15 [36-38] is a new data set that reflects real modern normal activities and contains synthetic contemporary attacks. This data set is completely different from NSL-KDD, which reflects a more modern and complex threat environment. The raw network packet of the UNSW-NB15 data set was created by the Tcpdump tool, then 49 features with the class label are generated by Argus, Bro-IDS tool and 12 algorithms [38]. The full dataset contains a total of 25,400,443 records. The partition of the full dataset are divided into a training set and a test set according to the hierarchical sampling method, namely, UNSW_NB15_training-set.csv and UNSW_NB15_testing-set.csv. The training dataset consists of 175,341 records whereas the testing dataset contains 82,332 records. The number of features in the partitioned dataset [37] is different from the number of features in the full dataset [36]. The partitioned data set has only 43 features with the class label, removing 6 features (i.e., dstip, srcip, sport, dsport, Ltime and Stime) from the full dataset. The partitioned dataset contains ten categories, one normal and nine attacks, namely, generic, exploits, fuzzers, DoS, reconnaissance, analysis, backdoor, shellcode and worms. Table 3 shows in detail the class distribution of the UNSW-NB15 dataset.

Table 3. The class distribution of the UNSW-NB15 dataset.

\begin{tabular}{lcc}
\hline \multirow{2}{*}{ Category } & Training Dataset & Testing Dataset \\
\cline { 2 - 3 } & UNSW_NB15_Training-Set & UNSW_NB15_Testing-Set \\
\hline Normal & 56,000 & 37,000 \\
Generic & 40,000 & 18,871 \\
Exploits & 33,393 & 11,132 \\
Fuzzers & 18,184 & 6062 \\
DoS & 12,264 & 4089 \\
Reconnaissance & 10,491 & 3496 \\
Analysis & 2000 & 677 \\
Backdoor & 1746 & 583 \\
Shellcode & 1133 & 378 \\
Worms & 130 & 44 \\
\hline Total & 175,341 & 82,332 \\
\hline
\end{tabular}

\subsection{Experimental Setup}

Our experiments were carried out to evaluate the performance of the proposed model. We used three different datasets from NSL-KDD, and UNSW-NB15 datasets. We compared the results of the proposed model with other well-known detection methods. The proposed system was implemented in the TensorFlow environment on the ThinkStation with 64 GB RAM, Intel E5-2620 CPU and 64-bit Windows 10 operating system. An appropriate number of hidden layers can improve the generalization performance of the DNN classifier. Since the number of input units is less than 200, according to empirical experience, the candidate number of hidden layers is $\{3,4\}$. DNN has the function of automatically extracting features, so the number of hidden units is set in a decreasing manner with the value of \{two times the number of categories, four times the number of categories, eight times the number of categories, more than 8 times the number of categories\}. When the learning rate is too large, the network will oscillate during training, resulting in no convergence. In TensorFlow, the default learning rate of the Adam optimizer is 0.001 , which I reduced by $50 \%$, so my candidate learning rate is $\left\{1 \times 10^{-3}, 5 \times 10^{-4}, 1 \times 10^{-4}\right\}$. L2 is used to avoid over-fitting issue. Here, if L2 is zero then we get back the original model. However, if L2 is very large, it will add too much weight and will lead to under-fitting, so my candidate L2 is $\left\{1 \times 10^{-4}, 1 \times 10^{-5}\right\}$. In order to overcome the vanishing gradient problem caused by Sigmoid or the explosion gradient problem caused by ReLU, we consider ReLU6 [50] as the activation function of hidden Layers. The parameters of the ICVAE-DNN network configuration are searched according to the following principles, as follows: 
- Number of hidden layers $=\{3,4\}$

- Number of hidden units $=\{$ two times the number of categories, four times the number of categories, eight times the number of categories, more than eight times the number of categories\}

- $\quad$ Learning rate $=\left\{1 \times 10^{-3}, 5 \times 10^{-4}, 1 \times 10^{-4}\right\}$

- $\mathrm{L} 2=\left\{1 \times 10^{-4}, 1 \times 10^{-5}\right\}$

- Activation function of hidden Layers $=\{$ ReLU6 $\}$

Grid search and three-fold cross-validation experiments are performed to find the optimal hyperparameters of a model which results in the most accurate predictions. The grid search traverses each group of hyperparameters in the search hyperparameter space. For each group of hyperparameters, three-fold cross-validation is used to evaluate. Three-fold cross-validation divides the original training dataset into three subsets, each of which shares the same proportion of each class of data. In each run of the model, two subsets are used to train the model and the remaining subset is used for test the model. By running the model three times, each subset of data has an equal chance to be used in testing part, and then the score of accuracy is computed by taking the average of the accuracy of the model on the testing subsets. Finally, the parameters that get the best cross-validation score are taken as the optimal parameter. The optimal network structures of the proposed model on the NSL-KDD and UNSW-NB15 data sets are 122-80-40-20-10-5 and 196-140-80-40-20-10, respectively. In the ICVAE encoder, the activation function of all hidden layers is ReLU6 [50], and the activation function of the output layer is linear. In the ICVAE decoder, the activation function of all hidden layers is ReLU6 [50], and the activation function of the output layer is Sigmoid. In the DNN, the activation function of all hidden layers is ReLU6 [50], and the activation function of the output layer is Softmax. The learning rate of ICVAE is $5 \times 10^{-4}$, the learning rate of DNN is $1 \times 10^{-4}$, the value of L2 regularization is $1 \times 10^{-4}$, and the optimization algorithm is Adam [49]. Based on these optimal parameters, the training charts of ICVAE-DNN are shown in Figures 5 and 6 . As can be seen from Figures $5 \mathrm{~b}$ and $6 \mathrm{~b}$, the initial loss of DNN was relatively low, which implies that after initializing the weight of DNN hidden layers with ICVAE encoder, DNN was close to global optimum. From the average loss of ICVAE and DNN and the accuracy of the training data, it can be seen that the network is basically convergent.

We performed performance comparisons from two aspects: oversampling method and classification method. Tables 4-8 show the comparison results for different oversampling methods. Tables 9-11 show the performance comparison between the ICVAE-DNN and six well-known models. In addition, the detection performance of the ICVAE-DNN is further compared with other state-of-the-art models. Table 12 depicts the comparison results on the NSL-KDD (KDDTest+), NSL-KDD (KDDTest-21), and UNSW-NB15 datasets.

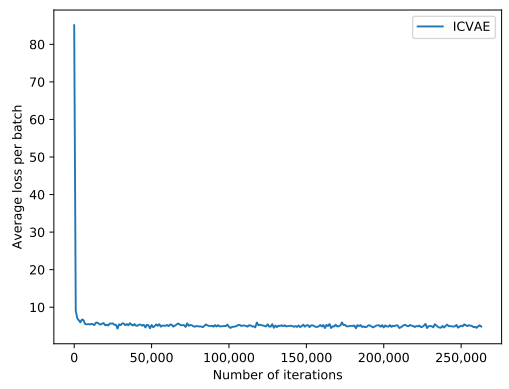

(a) ICVAE average loss

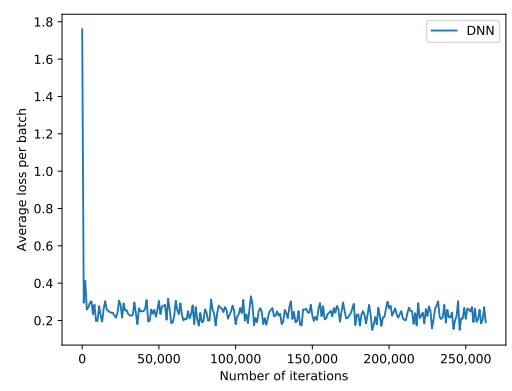

(b) DNN average loss

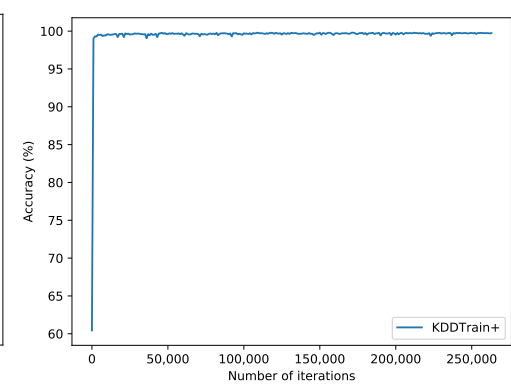

(c) Accuracy of training set

Figure 5. Training charts on the NSL-KDD dataset. 


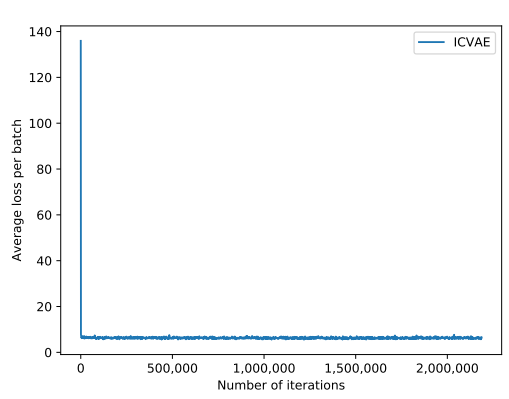

(a) ICVAE average loss

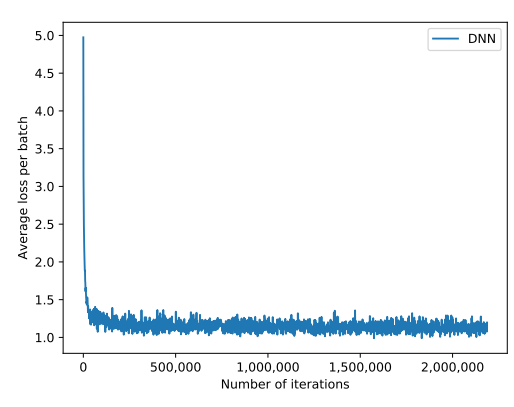

(b) DNN average loss

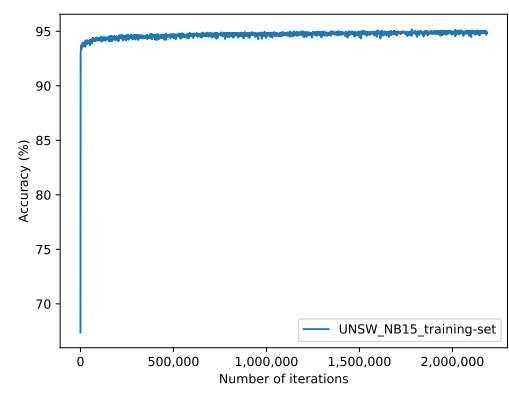

(c) Accuracy of training set

Figure 6. Training charts on the UNSW-NB15 dataset.

\subsection{Results and Discussion}

\subsubsection{The Detection Performance}

As is evident from Tables 2 and 3, the training samples are imbalanced on the NSL-KDD and UNSW-NB15 datasets. The U2R and R2L have minority records on the NSL-KDD dataset, and the worms and shellcode have minority records on the UNSW-NB15 dataset. We use ICVAE decoder to generate several records of the specified category to balance the training data, and the results are shown in Tables 4 and 5 .

Table 4. Number of samples generated on the NSL-KDD training dataset.

\begin{tabular}{lccc}
\hline Category & Number of Original Records & Number of Newly Generated Records & Total \\
\hline Normal & 13,449 & 0 & 13,449 \\
Probe & 2289 & 11,160 & 13,449 \\
DoS & 9234 & 4215 & 13,449 \\
U2R & 11 & 13,438 & 13,449 \\
R2L & 209 & 13,240 & 13,449 \\
\hline Total & 25,192 & 42,053 & 67,245 \\
\hline
\end{tabular}

Table 5. Number of samples generated on the UNSW-NB15 training dataset.

\begin{tabular}{lccc}
\hline Category & Number of Original Records & Number of Newly Generated Records & Total \\
\hline Normal & 56,000 & 0 & 56,000 \\
Generic & 40,000 & 16,000 & 56,000 \\
Exploits & 33,393 & 22,607 & 56,000 \\
Fuzzers & 18,184 & 37,816 & 56,000 \\
DoS & 12,264 & 43,736 & 56,000 \\
Reconnaissance & 10,491 & 45,509 & 56,000 \\
Analysis & 2000 & 54,000 & 56,000 \\
Backdoor & 1746 & 54,254 & 56,000 \\
Shellcode & 1133 & 54,867 & 56,000 \\
Worms & 130 & 55,870 & 56,000 \\
\hline Total & 175,341 & 384,659 & 560,000 \\
\hline
\end{tabular}

The proposed ICVAE-DNN used the ICVAE decoder to synthesize minority attack samples. The most popular oversampling methods used to synthesize minority attack samples are random over sampler (ROS) [53], SMOTE [54], and ADASYN [55]. In order to demonstrate the superiority of ICVAE-DNN in oversampling technology, three classification models are constructed based on three oversampling methods, namely ROS-DNN, SMOTE-DNN and ADASYN-DNN. Tables 6-8 show the comparison results. 
Table 6. Comparison of detection performance for different oversampling methods on the NSL-KDD (KDDTest+) data set (\%).

\begin{tabular}{lcccccccccc}
\hline Model & Normal & Probe & DoS & U2R & R2L & Accuracy & Recall & Precision & F1-Score & FPR \\
\hline ROS-DNN & 92.61 & 56.26 & 80.32 & 6.00 & 12.75 & 78.26 & 67.41 & 92.34 & 77.93 & 7.39 \\
SMOTE-DNN & 96.59 & 56.75 & 82.19 & $\mathbf{1 1 . 0 0}$ & 10.93 & 81.16 & 69.48 & 96.42 & 80.76 & 3.41 \\
ADASYN-DNN & 96.43 & 59.81 & 83.28 & 8.00 & 9.84 & 80.10 & 67.74 & 96.16 & 79.49 & 3.57 \\
ICVAE-DNN & $\mathbf{9 7 . 2 6}$ & $\mathbf{7 4 . 9 7}$ & $\mathbf{8 5 . 6 5}$ & $\mathbf{1 1 . 0 0}$ & $\mathbf{4 4 . 4 1}$ & $\mathbf{8 5 . 9 7}$ & $\mathbf{7 7 . 4 3}$ & $\mathbf{9 7 . 3 9}$ & $\mathbf{8 6 . 2 7}$ & $\mathbf{2 . 7 4}$ \\
\hline
\end{tabular}

Table 7. Comparison of detection performance for different oversampling methods on the NSL-KDD (KDDTest-21) data set (\%).

\begin{tabular}{lcccccccccc}
\hline Model & Normal & Probe & DoS & U2R & R2L & Accuracy & Recall & Precision & F1-Score & FPR \\
\hline ROS-DNN & 85.83 & 65.36 & 74.14 & 5.50 & 10.02 & 63.43 & 58.46 & 94.89 & 72.35 & 14.17 \\
SMOTE-DNN & 86.76 & 60.99 & 66.86 & 12.00 & 14.45 & 65.34 & 60.59 & 95.37 & 74.10 & 13.24 \\
ADASYN-DNN & 67.98 & 54.29 & 67.94 & 8.00 & 11.58 & 57.76 & 55.50 & 88.65 & 68.26 & 32.02 \\
ICVAE-DNN & $\mathbf{8 7 . 0 4}$ & $\mathbf{7 9 . 8 9}$ & $\mathbf{7 7 . 8 7}$ & $\mathbf{1 1 . 5 0}$ & $\mathbf{2 3 . 1 7}$ & $\mathbf{7 5 . 4 3}$ & $\mathbf{7 2 . 8 6}$ & $\mathbf{9 6 . 2 0}$ & $\mathbf{8 2 . 9 2}$ & $\mathbf{1 2 . 9 6}$ \\
\hline
\end{tabular}

Table 8. Comparison of detection performance for different oversampling methods on the UNSW-NB15 dataset $(\%)$.

\begin{tabular}{lcccc}
\hline Class & ROS-DNN & SMOTE-DNN & ADASYN-DNN & ICVAE-DNN \\
\hline Normal & 57.26 & 57.66 & 57.29 & $\mathbf{8 0 . 9 9}$ \\
Generic & 95.94 & 95.38 & 96.22 & $\mathbf{9 6 . 3 1}$ \\
Exploits & 49.69 & 50.59 & 44.36 & $\mathbf{7 1 . 0 2}$ \\
Fuzzers & 56.88 & 58.99 & 59.07 & 35.35 \\
DoS & 10.00 & $\mathbf{2 2 . 6 5}$ & 2.52 & 7.92 \\
Reconnaissance & 48.17 & $\mathbf{8 3 . 8 1}$ & 47.63 & 80.29 \\
Analysis & 13.44 & 15.36 & $\mathbf{8 6 . 5 6}$ & 15.21 \\
Backdoor & $\mathbf{4 9 . 4 0}$ & 42.20 & 0.86 & 20.58 \\
Shellcode & 90.74 & 84.39 & 80.69 & $\mathbf{9 1 . 8 0}$ \\
Worms & 34.09 & 52.27 & 47.73 & $\mathbf{7 9 . 5 5}$ \\
\hline Accuracy & 80.52 & 80.92 & 80.72 & $\mathbf{8 9 . 0 8}$ \\
Recall (DR) & 99.50 & $\mathbf{9 9 . 9 0}$ & 99.85 & 95.68 \\
Precision & 74.04 & 74.30 & 74.12 & $\mathbf{8 6 . 0 5}$ \\
F1-score & 84.90 & 85.22 & 85.08 & $\mathbf{9 0 . 6 1}$ \\
FPR & 42.74 & 42.34 & 42.71 & $\mathbf{1 9 . 0 1}$ \\
\hline
\end{tabular}

As can be seen from Tables 6 and 7, the proposed ICVAE-DNN achieved the best detection performance on the NSL-KDD (KDDTest+) and NSL-KDD (KDDTest-21) data sets. Table 8 shows that the proposed ICVAE-DNN has a higher detection rate compared to all over-sampling methods except for ROS-DNN (only in backdoor attack), SMOTE-DNN (in DoS and reconnaissance attacks) and ADASYN-DNN (in fuzzers and analysis attacks) on the UNSW-NB15 dataset. ROS-DNN has a better detection rate in backdoor class compared to ICVAE-DNN (28.82\% more), SMOTE-DNN shows a comparable detection rate to ICVAE-DNN in classes DoS and reconnaissance $(14.73 \%$ and $3.52 \%$ more, respectively), and the detection rate of ADASYN-DNN in fuzzers and analysis attacks is $23.72 \%$ and $71.35 \%$ higher than that of our model, respectively. However, compared to three well-known oversampling methods, ICVAE-DNN has higher overall accuracy, precision, F1-score and FPR. These reasons may be due to defects in the three oversampling techniques of ROS, SMOTE and ADASYN. ROS-DNN is a simple copy of the training sample, which easily leads to model overfitting problems and reduces the generalization performance of the classifier. SMOTE-DNN uses the nearest neighbor method to generate new samples for each minority sample, which is prone to over-generalization. ADASYN-DNN uses the $\Gamma$ distribution to automatically determine the number of samples that need to be synthesized for each minority sample, which are susceptible to outliers and cause changes in the spatial distribution of the original samples. ICVAE uses the spatial distribution of latent variables to generate samples under the guidance of class labels, and uses the reconstruction 
error to filter the generated samples to ensure that the generated samples are more consistent with the spatial distribution of the original samples. In addition, the trained ICVAE encoder was used to initialize the weight of the hidden layers of the DNN classifier, which made it easier for the DNN classifier to achieve global optimization, thereby improving classification performance. It was also demonstrated from Tables 6-8 that the proposed ICVAE is more suitable for solving the classification problem of imbalanced samples.

We compared the results of ICVAE-DNN with some well-known classification methods such as KNN (K-Nearest Neighbor), MultinomialNB (multinomial naive Bayes), SVM, RF, DNN, and DBN. We perform performance evaluation based on the five metrics introduced in Section 5.1. The results compared with some well-known classifiers are depicted in Tables 9-11.

Table 9. Comparison of detection performance for different classification methods on the NSL-KDD (KDDTest+) dataset (\%).

\begin{tabular}{lcccccccccc}
\hline Model & Normal & Probe & DoS & U2R & R2L & Accuracy & Recall & Precision & F1-Score & FPR \\
\hline KNN & 92.78 & 59.4 & 82.25 & 3.50 & 3.56 & 76.51 & 64.19 & 92.16 & 75.68 & 7.22 \\
MultinomialNB & 96.03 & $\mathbf{8 2 . 6 1}$ & 37.1 & 0.50 & 22.22 & 78.73 & 65.64 & 95.62 & 77.85 & 3.97 \\
RF & $\mathbf{9 7 . 3 7}$ & 58.53 & 80.24 & 0.50 & 7.55 & 76.49 & 60.69 & 96.84 & 74.62 & $\mathbf{2 . 6 3}$ \\
SVM & 92.82 & 61.71 & 74.85 & 0.00 & 0.00 & 72.28 & 56.73 & 91.26 & 69.97 & 7.18 \\
DNN & 96.10 & 65.30 & 85.40 & 2.50 & 14.56 & 80.22 & 68.21 & 95.85 & 79.70 & 3.90 \\
DBN & 97.04 & 69.85 & 83.11 & 5.50 & 12.56 & 80.82 & 68.53 & 96.84 & 80.26 & 2.96 \\
ICVAE-DNN & 97.26 & 74.97 & $\mathbf{8 5 . 6 5}$ & $\mathbf{1 1 . 0 0}$ & $\mathbf{4 4 . 4 1}$ & $\mathbf{8 5 . 9 7}$ & $\mathbf{7 7 . 4 3}$ & $\mathbf{9 7 . 3 9}$ & $\mathbf{8 6 . 2 7}$ & 2.74 \\
\hline
\end{tabular}

As can be seen from Table 9, ICVAE-DNN had the highest overall accuracy, recall, precision and F1-score on NSL-KDD (KDDTest+) data set than all well-known classifiers, except RF (with $0.11 \%$ higher in FPR). Moreover, compared with other classifiers, ICVAE-DNN has a higher detection rate in DOS, U2R and R2L classes. RF has a slightly higher detection rate in the normal class compared to ICVAE-DNN (0.11\% more), and MultinomialNB has a $7.64 \%$ higher detection rate in Probe class than ICVAE-DNN. However, ICVAE-DNN has the highest overall detection rate. In addition, ICVAE-DNN achieves the highest detection rates in two important minority U2R and R2L attacks, indicating that ICVAE-DNN is more effective in detecting minority attacks and unknown attacks.

Table 10. Comparison of detection performance for different classification methods on the NSL-KDD (KDDTest-21) dataset (\%).

\begin{tabular}{lcccccccccc}
\hline Model & Normal & Probe & DoS & U2R & R2L & Accuracy & Recall & Precision & F1-Score & FPR \\
\hline KNN & 68.49 & 59.08 & 69.81 & 3.50 & 3.56 & 55.50 & 52.62 & 88.27 & 65.93 & 31.51 \\
MultinomialNB & 83.32 & $\mathbf{8 2 . 8 1}$ & 38.12 & 0.50 & 22.22 & 60.08 & 54.93 & 93.69 & 69.25 & 16.68 \\
RF & $\mathbf{8 8 . 3 8}$ & 60.45 & 66.08 & 0.50 & 10.42 & 56.84 & 49.84 & 95.08 & 65.39 & $\mathbf{1 1 . 6 2}$ \\
SVM & 68.26 & 61.41 & 56.79 & 0.00 & 0.00 & 47.38 & 42.74 & 85.85 & 57.07 & 31.74 \\
DNN & 86.29 & 67.86 & 64.30 & 4.50 & 13.94 & 60.96 & 55.34 & 94.79 & 69.88 & 13.71 \\
DBN & 71.75 & 58.33 & 71.72 & 0.50 & 13.25 & 57.45 & 54.28 & 89.65 & 67.62 & 28.25 \\
ICVAE-DNN & 87.04 & 79.89 & $\mathbf{7 7 . 8 7}$ & $\mathbf{1 1 . 5 0}$ & $\mathbf{2 3 . 1 7}$ & $\mathbf{7 5 . 4 3}$ & $\mathbf{7 2 . 8 6}$ & $\mathbf{9 6 . 2 0}$ & $\mathbf{8 2 . 9 2}$ & 12.96 \\
\hline
\end{tabular}

Table 10 shows that ICVAE-DNN achieves the best overall performance, except for RF (only with $1.34 \%$ difference in the overall FPR). The detection rate of RF in the Normal class is $1.34 \%$ higher than that of ICVAE-DNN. In the probe attack, MultinomialNB achieved a 2.92\% higher detection rate than ICVAE-DNN. However, RF and MultinomialNB were poor in other performances. ICVAE-DNN had the highest detection rates in minority classes U2R and R2L. Tables 9 and 10 show that all classifiers have very low detection rates in the U2R and R2L attacks. The main reason was that there were too few U2R and R2L attacks in the training data set (with 11 and 209 samples, respectively). As can be seen from Table 2, almost half of the U2R and R2L attacks in the testing data set never appeared in the training data set, such as httptunnel, snmpgetattack, snmpguess, etc. As a result, all classifiers are not fully trained. Moreover, some attacks in the R2L class, such as sendmail and snmpguess attacks, 
exhibit features that were highly similar to normal records, which can cause the classifier to misclassify them as normal records.

Table 11 shows that ICVAE-DNN achieves the best overall performance compared to the other six well-known models, except for DBN in the overall recall (slightly higher by 3.22\%). ICVAE-DNN reaches the highest detection rate in classes normal, reconnaissance, analysis, backdoor, shellcode and worms. MultinomialNB achieves the highest detection rate of $70.11 \%$ in the DoS class, which implies that the DoS attack features conform to the polynomial distribution. KNN achieved the highest detection rate of $96.63 \%$ in the generic class, but the overall DR of KNN was $1.67 \%$ lower than that of ICVAE-DNN. In fuzzers attacks, SVM achieved a 39.66\% higher detection rate than ICVAE-DNN. The detection rate of DBN in exploits attack was $16.4 \%$ higher than that of our model, and the overall detection rate was also $3.22 \%$ higher, but its overall F1-score was $2.16 \%$ lower. However, ICVAE-DNN achieved the highest detection rates in minority and important attacks: analysis, backdoor, shellcode and worms. For example, the detection rate of ICVAE-DNN in the worms class was $68.44 \%$ and $79.55 \%$ higher than that of KNN and SVM, respectively. In addition, we can see that all classifiers have low detection rate in classes analysis and backdoor, mainly because the analysis and backdoor attack features are highly similar to exploits attack features. As a result, classifiers misclassify most of the analysis and backdoor attacks as exploits attacks.

Table 11. Comparison of detection performance for different classification methods on the UNSW-NB15 dataset $(\%)$.

\begin{tabular}{lccccccc}
\hline Class & KNN & MultinomialNB & RF & SVM & DNN & DBN & ICVAE-DNN \\
\hline Normal & 74.56 & 57.78 & 76.42 & 57.64 & 74.31 & 69.68 & $\mathbf{8 0 . 9 9}$ \\
Generic & $\mathbf{9 6 . 6 3}$ & 96.29 & 96.73 & 96.24 & 96.41 & 96.34 & 96.31 \\
Exploits & 74.48 & 42.05 & 76.24 & 74.51 & 86.20 & $\mathbf{8 7 . 4 2}$ & 71.02 \\
Fuzzers & 42.33 & 42.48 & 53.33 & $\mathbf{7 5 . 0 1}$ & 45.53 & 55.10 & 35.35 \\
DoS & 19.44 & $\mathbf{7 0 . 1 1}$ & 10.37 & 0.00 & 7.65 & 8.24 & 7.92 \\
Reconnaissance & 58.94 & 36.76 & 78.52 & 0.57 & 77.46 & 79.81 & $\mathbf{8 0 . 2 9}$ \\
Analysis & 1.48 & 0.00 & 5.17 & 0.00 & 0.59 & 0.00 & $\mathbf{1 5 . 2 1}$ \\
Backdoor & 2.56 & 0.00 & 11.49 & 0.00 & 8.06 & 0.34 & $\mathbf{2 0 . 5 8}$ \\
Shellcode & 14.47 & 0.00 & 60.85 & 0.00 & 60.32 & 59.26 & $\mathbf{9 1 . 8 0}$ \\
Worms & 11.11 & 0.00 & 4.55 & 0.00 & 36.36 & 0.00 & $\mathbf{7 9 . 5 5}$ \\
\hline Accuracy & 85.38 & 76.14 & 87.45 & 78.91 & 86.95 & 85.77 & $\mathbf{8 9 . 0 8}$ \\
Recall (DR) & 94.01 & 91.12 & 96.46 & 96.27 & 97.28 & $\mathbf{9 8 . 9 0}$ & 95.68 \\
Precision & 82.05 & 72.56 & 83.36 & 73.58 & 82.26 & 79.99 & $\mathbf{8 6 . 0 5}$ \\
F1-score & 87.63 & 80.79 & 89.44 & 83.41 & 89.14 & 88.45 & $\mathbf{9 0 . 6 1}$ \\
FPR & 25.19 & 42.22 & 23.58 & 42.36 & 25.69 & 30.32 & $\mathbf{1 9 . 0 1}$ \\
\hline
\end{tabular}

\subsection{Additional Comparison}

To better demonstrate the performance of ICVAE-DNN, we compare its performance with nine state-of-the-art intrusion detection methods, namely, SCDNN (spectral clustering and deep neural network) [44], STL (self-taught learning) [56], DNN [57], Gaussian-Bernoulli RBM [18], RNN-IDS [58], CASCADE-ANN (a multiclass cascade of artificial neural network) [59], ID-CVAE (intrusion detection CVAE) [16], EM Clustering and DT [38]. Table 12 demonstrates the comparison results of ICVAE-DNN proposed on three datasets with other models in terms of accuracy, DR and FPR. Compared with other methods on the NSL-KDD (KDDTest+) and NSL-KDD (KDDTest-21) datasets, the proposed method achieved the best performance in terms of accuracy, DR and FPR. As shown in Table 12, ICVAE-DNN achieved the highest accuracy of $89.08 \%$ and DR of $95.68 \%$ on the UNSW-NB15 data set, but its FPR is slightly worse. The CASCADE-ANN proposed by Baig et al. [59] achieved a lower FPR (with 5.91\% less) than ICVAE-DNN, but its accuracy and DR were worse. The experimental results show that ICVAE-DNN had higher accuracy, DR and FPR than other state-of-the-art intrusion detection methods except CASCADE-ANN (FPR on UNSW-NB15 dataset). Based on the experimental results, we concluded that ICVAE-DNN has better detection performance for network intrusion detection. 
Table 12. Comparison results based on NSL-KDD and UNSW-NB15 datasets (N/A means no available results, * Ranked first, ${ }^{* *}$ Ranked second).

\begin{tabular}{|c|c|c|c|c|}
\hline Method & Dataset & Accuracy (\%) & DR (\%) & FPR $(\%)$ \\
\hline SCDNN [44] & NSL-KDD (KDDTest+) & 72.64 & 57.48 & $\mathrm{~N} / \mathrm{A}$ \\
\hline STL [56] & NSL-KDD (KDDTest+) & 74.38 & $62.99 * *$ & $7.21^{* *}$ \\
\hline DNN [57] & NSL-KDD (KDDTest+) & 75.75 & $\mathrm{~N} / \mathrm{A}$ & $\mathrm{N} / \mathrm{A}$ \\
\hline Gaussian-Bernoulli RBM [18] & NSL-KDD (KDDTest+) & 73.23 & $\mathrm{~N} / \mathrm{A}$ & $\mathrm{N} / \mathrm{A}$ \\
\hline RNN-IDS [58] & NSL-KDD (KDDTest+) & $81.29^{* *}$ & $\mathrm{~N} / \mathrm{A}$ & $\mathrm{N} / \mathrm{A}$ \\
\hline ID-CVAE [16] & NSL-KDD (KDDTest+) & 80.10 & $\mathrm{~N} / \mathrm{A}$ & $\mathrm{N} / \mathrm{A}$ \\
\hline ICVAE-DNN & NSL-KDD (KDDTest+) & $85.97 *$ & 77.43 * & 2.74 * \\
\hline SCDNN [44] & NSL-KDD (KDDTest-21) & 44.55 & 37.85 & $\mathrm{~N} / \mathrm{A}$ \\
\hline STL [56] & NSL-KDD (KDDTest-21) & 57.34 & $52.73^{* *}$ & $15.06^{* *}$ \\
\hline RNN-IDS [58] & NSL-KDD (KDDTest-21) & $64.67^{* *}$ & $\mathrm{~N} / \mathrm{A}$ & $\mathrm{N} / \mathrm{A}$ \\
\hline ICVAE-DNN & NSL-KDD (KDDTest-21) & $75.43 *$ & $72.86 *$ & $12.96 *$ \\
\hline CASCADE-ANN [59] & UNSW-NB15 & $86.40^{* *}$ & $86.74^{* *}$ & 13.10 * \\
\hline EM Clustering [38] & UNSW-NB15 & 78.47 & $\mathrm{~N} / \mathrm{A}$ & $\mathrm{N} / \mathrm{A}$ \\
\hline DT [38] & UNSW-NB15 & 85.56 & $\mathrm{~N} / \mathrm{A}$ & $\mathrm{N} / \mathrm{A}$ \\
\hline ICVAE-DNN & UNSW-NB15 & 89.08 * & $95.68 *$ & $19.01 * *$ \\
\hline
\end{tabular}

\section{Conclusions}

In this paper, we propose a novel intrusion detection approach called ICVAE-DNN that combines the ICVAE with DNN. For large data sets, ICVAE can learn and explore the potential sparse representations between network data features and categories. The trained ICVAE encoder is used to initialize the weight of DNN hidden layers. DNN can learn faster and easier than traditional multi-layer perceptron networks, thus avoiding stopping in the local minima. The ICVAE decoder is able to generate various unknown attack samples according to the specified intrusion categories, which not only balances the training data set, but also increases the diversity of training samples, so ICVAE-DNN can improve the detection rate of minority attacks and unknown attacks. DNN can automatically extract high-level abstract features from the training data, thus it can reduce data dimension to avoid dimension curse. DNN integrates feature extraction and classification methods into a system that automatically extracts features and performs classification without a lot of heuristic rules and manual experience. The classification performance of ICVAE-DNN is evaluated on the NSL-KDD (KDDTest+), NSL-KDD (KDDTest-21), and UNSW-NB15 datasets and compared with six well-known classifiers. Moreover, the experimental results show that the proposed ICVAE-DNN provides higher detection rates in minority attacks (i.e., U2R, R2L, shellcode and worms) than the six well-known classification algorithms: KNN, MultinomialNB, RF, SVM, DNN and DBN. In addition, compared with the state-of-the-art classifiers (such as SCDNN, STL, DNN, Gaussian-Bernoulli RBM, RNN-IDS, ID-CVAE, CASCADE-ANN, EM Clustering and DT), the proposed ICVAE-DNN achieves higher accuracy, detection rate and false positive rate. These experiments prove that ICVAE-DNN is more suitable for detecting network intrusion, especially for minority attacks and unknown attacks.

Considering future work, we plan to study an effective way to improve the detection performance of minority attacks and unknown attacks. We plan to use the adversarial learning method to explore the spatial distribution of ICVAE latent variables to better reconstruct input samples. Through the adversarial learning method, similar minority attacks can be synthesized, and the diversity of training samples can be increased. As a result, the detection performance of the ICVAE-DNN can be further improved.

Author Contributions: conceptualization, Y.Y. (Yanqing Yang), K.Z. and C.W.; methodology, Y.Y. (Yanqing Yang), K.Z., C.W. and Y.Y. (Yixian Yang); software, Y.Y. (Yanqing Yang); validation, Y.Y. (Yanqing Yang), K.Z. and C.W.; formal analysis, K.Z. and Y.Y. (Yixian Yang); investigation, Y.Y. (Yanqing Yang) and K.Z.; writing一original draft preparation, Y.Y. (Yanqing Yang); writing - review and editing, Y.Y. (Yanqing Yang) and K.Z.; visualization, Y.Y. (Yanqing Yang); supervision, K.Z. and C.W. and Y.Y. (Yanqing Yang); project administration, K.Z. and C.W. 
Funding: This research was supported by the National Key R\&D Program of China (2017YFB0802703) and National Natural Science Foundation of China (61602052).

Acknowledgments: The authors would like to thank the anonymous reviewers for their contribution to this paper. Conflicts of Interest: The authors declare no conflict of interest.

\section{References}

1. Lin, J.C.W.; Wu, J.M.T.; Fournier-Viger, P.; Djenouri, Y.; Chen, C.H.; Zhang, Y. A Sanitization Approach to Secure Shared Data in an IoT Environment. IEEE Access 2019, 7, 25359-25368. [CrossRef]

2. Zhang, Y.; Li, P.; Wang, X. Intrusion Detection for IoT Based on Improved Genetic Algorithm and Deep Belief Network. IEEE Access 2019, 7, 31711-31722. [CrossRef]

3. Background to IoT Security. Available online: https://safenet.gemalto.com/iot-2018/iot-security (accessed on 14 May 2019).

4. SecurityWeek. Available online: https://www.securityweek.com/top-dutch-banks-hit-cyber-attacks (accessed on 14 May 2019).

5. Winter Olympics Hit by Cyber-Attack. Available online: https://www.bbc.com/news/technology-43030673 (accessed on 14 May 2019).

6. Ali, M.H.; Al Mohammed, B.A.D.; Ismail, A.; Zolkipli, M.F. A new intrusion detection system based on fast learning network and particle swarm optimization. IEEE Access 2018, 6, 20255-20261. [CrossRef]

7. Aljawarneh, S.; Aldwairi, M.; Yassein, M.B. Anomaly-based intrusion detection system through feature selection analysis and building hybrid efficient model. J. Comput. Sci. 2018, 25, 152-160. [CrossRef]

8. Kabir, E.; Hu, J.; Wang, H.; Zhuo, G. A novel statistical technique for intrusion detection systems. Fut. Gener. Comput. Syst. 2018, 79, 303-318. [CrossRef]

9. Karami, A. An anomaly-based intrusion detection system in presence of benign outliers with visualization capabilities. Expert Syst. Appl. 2018, 108, 36-60. [CrossRef]

10. Moustafa, N.; Creech, G.; Slay, J. Anomaly Detection System Using Beta Mixture Models and Outlier Detection. In Progress in Computing, Analytics and Networking; Springer: Berlin, Germany, 2018; pp. 125-135.

11. Tian, Y.; Mirzabagheri, M.; Bamakan, S.M.H.; Wang, H.; Qu, Q. Ramp loss one-class support vector machine; A robust and effective approach to anomaly detection problems. Neurocomputing 2018, 310, 223-235. [CrossRef]

12. Vijayanand, R.; Devaraj, D.; Kannapiran, B. Intrusion detection system for wireless mesh network using multiple support vector machine classifiers with genetic-algorithm-based feature selection. Comput. Secur. 2018, 77, 304-314. [CrossRef]

13. Ganeshan, R.; Paul Rodrigues, S. I-AHSDT: Intrusion detection using adaptive dynamic directive operative fractional lion clustering and hyperbolic secant-based decision tree classifier. J. Exp. Theor. Artif. Intell. 2018, 30, 887-910. [CrossRef]

14. Serpen, G.; Aghaei, E. Host-based misuse intrusion detection using PCA feature extraction and kNN classification algorithms. Intell. Data Anal. 2018, 22, 1101-1114. [CrossRef]

15. Shone, N.; Ngoc, T.N.; Phai, V.D.; Shi, Q. A deep learning approach to network intrusion detection. IEEE Trans. Emerg. Top. Comput. Intell. 2018, 2, 41-50. [CrossRef]

16. Lopez-Martin, M.; Carro, B.; Sanchez-Esguevillas, A.; Lloret, J. Conditional variational autoencoder for prediction and feature recovery applied to intrusion detection in iot. Sensors 2017, 17, 1967. [CrossRef] [PubMed]

17. Malaiya, R.K.; Kwon, D.; Kim, J.; Suh, S.C.; Kim, H.; Kim, I. An Empirical Evaluation of Deep Learning for Network Anomaly Detection. In Proceedings of the 2018 International Conference on Computing, Networking and Communications (ICNC), Maui, HI, USA, 5-8 March 2018; pp. 893-898.

18. Imamverdiyev, Y.; Abdullayeva, F. Deep Learning Method for Denial of Service Attack Detection Based on Restricted Boltzmann Machine. Big Data 2018, 6, 159-169. [CrossRef] [PubMed]

19. Huda, S.; Miah, S.; Yearwood, J.; Alyahya, S.; Al-Dossari, H.; Doss, R. A malicious threat detection model for cloud assisted internet of things (CoT) based industrial control system (ICS) networks using deep belief network. J. Parallel Distrib. Comput. 2018, 120, 23-31. [CrossRef]

20. Tamer Aldwairi, D.P.; Novotny, M.A. An evaluation of the performance of Restricted Boltzmann Machines as a model for anomaly network intrusion detection. Comput. Netw. 2018, 144, 111-119. [CrossRef] 
21. Yang, Y.; Zheng, K.; Wu, C.; Niu, X.; Yang, Y. Building an Effective Intrusion Detection System Using the Modified Density Peak Clustering Algorithm and Deep Belief Networks. Appl. Sci. 2019, 9, 238. [CrossRef]

22. Li, C.; Wang, J.; Ye, X. Using a Recurrent Neural Network and Restricted Boltzmann Machines for Malicious Traffic Detection. NeuroQuantology 2018, 16. [CrossRef]

23. Resende, P.A.A.; Drummond, A.C. A Survey of Random Forest Based Methods for Intrusion Detection Systems. ACM Comput. Surv. 2018, 51, 48. [CrossRef]

24. Yadahalli, S.; Nighot, M.K. Adaboost based parameterized methods for wireless sensor networks. In Proceedings of the 2017 International Conference On Smart Technologies For Smart Nation (SmartTechCon), Bangalore, India, 17-19 August 2017; pp. 1370-1374.

25. Roy, S.S.; Krishna, P.V.; Yenduri, S. Analyzing Intrusion Detection System: An ensemble based stacking approach. In Proceedings of the 2014 IEEE International Symposium on Signal Processing and Information Technology (ISSPIT), Noida, India, 15-17 December 2015; pp. 000307-000309.

26. Aburomman, A.A.; Reaz, M.B.I. A survey of intrusion detection systems based on ensemble and hybrid classifiers. Comput. Secur. 2017, 65, 135-152. [CrossRef]

27. Wongsuphasawat, K.; Smilkov, D.; Wexler, J.; Wilson, J.; Mané, D.; Fritz, D.; Krishnan, D.; Viégas, F.B.; Wattenberg, M. Visualizing dataflow graphs of deep learning models in TensorFlow. IEEE Trans. Vis. Comput. Graph. 2018, 24, 1-12. [CrossRef]

28. Wang, Z. Deep Learning-Based Intrusion Detection With Adversaries. IEEE Access 2018, 6, 38367-38384. [CrossRef]

29. Xin, Y.; Kong, L.; Liu, Z.; Chen, Y.; Li, Y.; Zhu, H.; Gao, M.; Hou, H.; Wang, C. Machine Learning and Deep Learning Methods for Cybersecurity. IEEE Access 2018, 6, 35365-35381. [CrossRef]

30. Ambusaidi, M.A.; He, X.; Nanda, P.; Tan, Z. Building an intrusion detection system using a filter-based feature selection algorithm. IEEE Trans. Comput. 2016, 65, 2986-2998. [CrossRef]

31. Kingma, D.P.; Welling, M. Auto-encoding variational bayes. arXiv 2013, arXiv:1312.6114.

32. Kingma, D.P.; Mohamed, S.; Rezende, D.J.; Welling, M. Semi-supervised learning with deep generative models. In Proceedings of the 27th International Conference on Neural Information Processing Systems, Montreal, QC, Canada, 8-13 December 2014; pp. 3581-3589.

33. Sohn, K.; Lee, H.; Yan, X. Learning structured output representation using deep conditional generative models. In Proceedings of the 28th International Conference on Neural Information Processing Systems, Montreal, QC, Canada, 7-12 December 2015; pp. 3483-3491.

34. UNB. NSL-KDD Dataset. Available online: https://www.unb.ca/cic/datasets/nsl.html (accessed on 20 January 2019).

35. Dhanabal, L.; Shantharajah, S. A study on NSL-KDD dataset for intrusion detection system based on classification algorithms. Int. J. Adv. Res. Comput. Commun. Eng. 2015, 4, 446-452.

36. ACCS. UNSW-NB15 Dataset. Available online: https:/ /www.unsw.adfa.edu.au/unsw-canberra-cyber/ cybersecurity / ADFA-NB15-Datasets/ (accessed on 20 January 2019).

37. Moustafa, N.; Slay, J. UNSW-NB15: A comprehensive data set for network intrusion detection systems (UNSW-NB15 network data set). In Proceedings of the 2015 Military Communications and Information Systems Conference (MilCIS), Canberra, Australia, 10-12 November 2015; pp. 1-6.

38. Moustafa, N.; Slay, J. The evaluation of Network Anomaly Detection Systems: Statistical analysis of the UNSW-NB15 data set and the comparison with the KDD99 data set. Inf. Secur. J. A Glob. Perspect. 2016, 25, 18-31. [CrossRef]

39. Kawachi, Y.; Koizumi, Y.; Harada, N. Complementary Set Variational Autoencoder for Supervised Anomaly Detection. In Proceedings of the 2018 IEEE International Conference on Acoustics, Speech and Signal Processing (ICASSP), Calgary, AB, Canada, 15-20 April 2018; pp. 2366-2370.

40. Sun, J.; Wang, X.; Xiong, N.; Shao, J. Learning Sparse Representation With Variational Auto-Encoder for Anomaly Detection. IEEE Access 2018, 6, 33353-33361. [CrossRef]

41. Chandy, S.E.; Rasekh, A.; Barker, Z.A.; Shafiee, M.E. Cyberattack Detection using Deep Generative Models with Variational Inference. arXiv 2018, arXiv:1805.12511.

42. Osada, G.; Omote, K.; Nishide, T. Network Intrusion Detection Based on Semi-supervised Variational Auto-Encoder. In European Symposium on Research in Computer Security; Springer: Berlin, Germany, 2017; pp. 344-361. 
43. Thing, V.L. IEEE 802.11 network anomaly detection and attack classification: A deep learning approach. In Proceedings of the 2017 IEEE Wireless Communications and Networking Conference (WCNC), San Francisco, CA, USA, 19-22 March 2017; pp. 1-6.

44. Ma, T.; Wang, F.; Cheng, J.; Yu, Y.; Chen, X. A hybrid spectral clustering and deep neural network ensemble algorithm for intrusion detection in sensor networks. Sensors 2016, 16, 1701. [CrossRef]

45. Tang, T.; Zaidi, S.A.R.; McLernon, D.; Mhamdi, L.; Ghogho, M. Deep Recurrent Neural Network for Intrusion Detection in SDN-based Networks. In Proceedings of the 2018 4th IEEE Conference on Network Softwarization and Workshops (NetSoft), Montreal, QC, Canada, 25-29 June 2018.

46. Muna, A.H.; Moustafa, N.; Sitnikova, E. Identification of malicious activities in industrial internet of things based on deep learning models. J. Inf. Secur. Appl. 2018, 41, 1-11.

47. Doersch, C. Tutorial on variational autoencoders. arXiv 2016, arXiv:1606.05908.

48. Shen, L.; Lin, Z.; Huang, Q. Relay backpropagation for effective learning of deep convolutional neural networks. In Proceedings of the European Conference on Computer Vision, Amsterdam, The Netherlands, 8-16 October 2016; Springer: Berlin, Germany, 2016; pp. 467-482.

49. Kingma, D.P.; Ba, J. Adam: A method for stochastic optimization. arXiv 2014, arXiv:1412.6980.

50. Krizhevsky, A.; Hinton, G. Convolutional Deep Belief Networks on Cifar-10. Unpublished work, 2010; 40p.

51. KDDCup. KDD Cup Dataset. Available online: http://kdd.ics.uci.edu/databases/kddcup99/kddcup99. html (accessed on 19 January 2019).

52. Tavallaee, M.; Bagheri, E.; Lu, W.; Ghorbani, A.A. A detailed analysis of the KDD CUP 99 data set. In Computational Intelligence for Security and Defense Applications; IEEE: Ottawa, ON, Canada, 2009; pp. 1-6.

53. Lemaître, G.; Nogueira, F.; Aridas, C.K. Imbalanced-learn: A python toolbox to tackle the curse of imbalanced datasets in machine learning. J. Mach. Learn. Res. 2017, 18, 559-563.

54. Chawla, N.V.; Bowyer, K.W.; Hall, L.O.; Kegelmeyer, W.P. SMOTE: Synthetic minority over-sampling technique. J. Artif. Intell. Res. 2002, 16, 321-357. [CrossRef]

55. He, H.; Bai, Y.; Garcia, E.A.; Li, S. ADASYN: Adaptive synthetic sampling approach for imbalanced learning. In Proceedings of the 2008 IEEE International Joint Conference on Neural Networks (IEEE World Congress on Computational Intelligence), Hong Kong, China, 1-8 June 2008; pp. 1322-1328.

56. Javaid, A.; Niyaz, Q.; Sun, W.; Alam, M. A deep learning approach for network intrusion detection system. In Proceedings of the 9th EAI International Conference on Bio-inspired Information and Communications Technologies (Formerly BIONETICS), New York, NY, USA, 3-5 December 2016; pp. 21-26.

57. Tang, T.A.; Mhamdi, L.; McLernon, D.; Zaidi, S.A.R.; Ghogho, M. Deep learning approach for network intrusion detection in software defined networking. In Proceedings of the 2016 International Conference on Wireless Networks and Mobile Communications (WINCOM), Fez, Morocco, 26-29 October 2016; pp. 258-263.

58. Yin, C.; Zhu, Y.; Fei, J.; He, X. A deep learning approach for intrusion detection using recurrent neural networks. IEEE Access 2017, 5, 21954-21961. [CrossRef]

59. Baig, M.M.; Awais, M.M.; El-Alfy, E.S.M. A multiclass cascade of artificial neural network for network intrusion detection. J. Intell. Fuzzy Syst. 2017, 32, 2875-2883. [CrossRef]

(C) 2019 by the authors. Licensee MDPI, Basel, Switzerland. This article is an open access article distributed under the terms and conditions of the Creative Commons Attribution (CC BY) license (http:/ / creativecommons.org/licenses/by/4.0/). 